\title{
Arterias Renales Múltiples
}

\author{
Multiple Renal Arteries
}

${ }^{*}$ Olave, E.; *Henríquez, J.; ${ }^{* *}$ Puelma, F.; ${ }^{* *}$ Cruzat, C. \& ${ }^{* *}$ Soto, A.

\begin{abstract}
OLAVE, E.; HENRÍQUEZ, J.; PUELMA, F.; CRUZAT, C. \& SOTO, A. Arterias renales mútiples. Int. J. Morphol., 25(4):927-930, 2007.
\end{abstract}
RESUMEN: El conocimiento de las variaciones de la irrigación renal es de mucha importancia debido al incremento de transplantes renales y cirugías de reconstrucción vascular. La literatura muestra que generalmente hay una arteria renal para cada riñón, sin embargo pueden observarse variaciones de origen y de número. Presentamos dos casos de arterias renales triples izquierdas, en muestras pertenecientes a la Unidad de Anatomía de la Facultad de Ciencias de la Salud, Universidad Católica del Maule, Talca. En uno de los casos, el origen de la arteria renal R1(superior) se efectuó a nivel de la parte inferior del origen de la arteria mesentérica superior, la que después de un trayecto de $30 \mathrm{~mm}$ se dividió en dos ramas menores de calibre similar, que ingresaron en el hilio renal por delante de la vena renal. La rama R2 (media) se originó $4 \mathrm{~mm}$ distal a la anterior, correspondiendo a una rama posterior que también ingresó en el hilio; la rama R3 (inferior) lo hizo 23 mm distal a la R2. Esta última penetró en el riñón inmediatamente por debajo de la incisura del seno renal. En el segundo caso, hay diferencias con respecto al anterior, ya que la rama R1 y la R3 penetraron en el hilio renal, en cambio la R2 tiene un trayecto descendente y penetra de forma similar al R3 del caso anterior. El conocimiento de estas arterias múltiples es necesario y es de importancia considerarlas durante la intervenciones quirúrgicas que involucren a este órgano.

\section{PALABRAS CLAVE: Anatomía; Riñón; Arterias.}

\section{INTRODUCCIÓN}

El aumento de intervenciones quirúrgicas en el riñón hace que la anatomía de sus arterias y ramas adquieran una gran importancia. En la mayoría de los individuos se encuentra una arteria renal a cada lado, la que se divide previamente a su ingreso en el hilio renal, en dos o más ramas, (Testut \& Latarjet, 1969; Dangelo \& Fattini, 1988; Williams et al., 1995; Moore \& Dalley, 2002; Khamanarong et al., 2004). Este padrón no está exento de variaciones, ya que se han descrito arterias renales dobles, uni o bilaterales (Tanyeli et al., 2006) o en menor porcentaje, tres arterias renales (Khamanarong et al.; Raheem et al., 2007).

Considerando la importancia de comunicar estas variaciones anatómicas, se presenta una inusual disposición de tres arterias renales.

\section{MATERIAL Y MÉTODO}

Las variaciones mencionadas se observaron en dos preparaciones anatómicas utilizadas en docencia en los La- boratorios de Anatomía de la Facultad de Ciencias de la Salud de la Universidad Católica del Maule, Talca, Chile.

Estas muestras corresponden a secciones de la porción abdominal de la aorta, vena cava inferior, el riñón correspondiente y sus vasos. Los elementos vasculares fueron pintados para una mejor demostración. Ambas variaciones se encontraron en el lado izquierdo.

En ellas se efectuó una descripción de las arterias y se obtuvieron algunos registros métricos de las distancias entre cada una de ellas y de su trayecto antes de dividirse. Para una mejor comprensión se denominó a la más superior como R1, a la media como R2 y a la más inferior como R3.

\section{DESCRIPCIÓN}

En uno de los casos (Fig.1), el origen de la arteria renal R1, se efectuó desde la porción abdominal de la aorta, a nivel de la parte inferior del origen de la arteria mesentérica

\footnotetext{
* Facultad de Medicina, Universidad de La Frontera, Temuco, Chile.

** Facultad de Ciencias de la Salud, Universidad Católica del Maule, Talca, Chile.

Post-graduación en Ciencias, Mención Morfología, Universidad de La Frontera, Chile.
} 
superior; esta R1 después de un trayecto de $30 \mathrm{~mm}$ se dividió en dos ramas menores de calibre similar, que ingresaron en el hilio renal por delante de la vena renal. La rama $\mathrm{R} 2$ se originó $4 \mathrm{~mm}$ distal a la anterior, correspondiendo a una rama posterior que también ingresó en el hilio y la rama R3 se originó $23 \mathrm{~mm}$ distal a la R2. Esta última penetró en el riñón inmediatamente por debajo del hilio renal. La vena renal recibía a la la vena gonadal correspondiente y era pre aórtica.

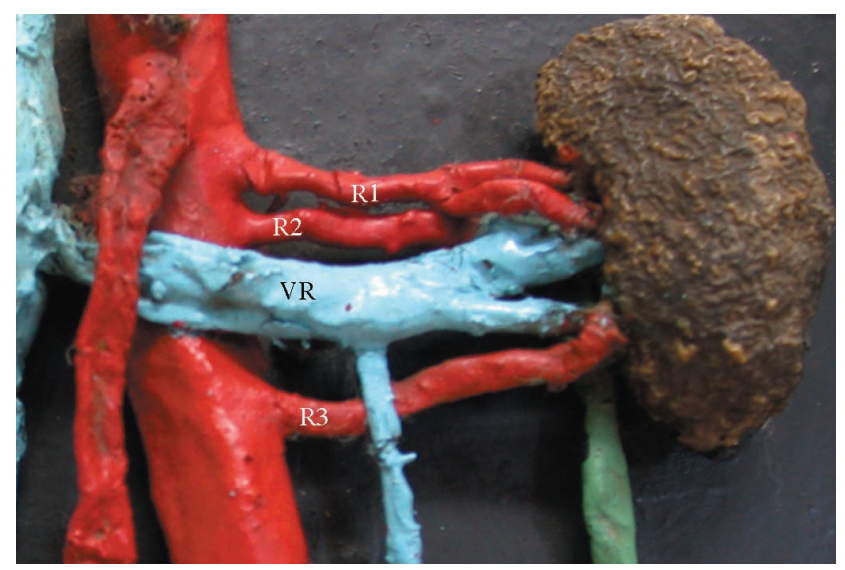

Fig. 1. Tres arterias renales, lado izquierdo (R1, R2 y R3). La vena renal (VR) se interponde entre las dos arterias superiores y la inferior.

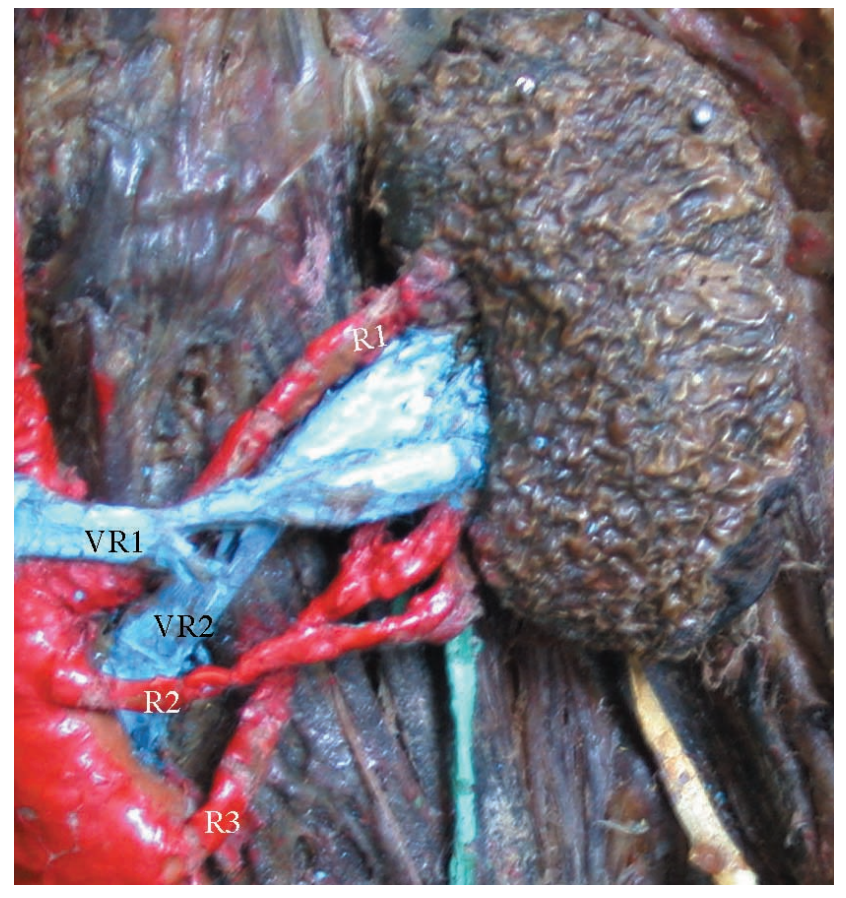

Fig. 2. Tres arterias renales en lado izquierdo (R1, R2, y R3). Se observa el drenaje venoso del riñón a través de venas con trayecto pre (VR1) y retro-aórtico (VR2).
En el segundo caso (Fig. 2), la rama R1 y la R3 se originaron de la parte lateral de la aorta abdominal y penetraron en el hilio renal, en cambio la R2 que se originó de la parte anterolateral de la aorta, tuvo un trayecto descendente y penetró en el riñón, por debajo del hilio renal. La R1 se originó $9 \mathrm{~mm}$ distal al origen de la arteria mesentérica superior; entre R1 y R2 se registraron $5 \mathrm{~mm}$ y entre R2 y R3 hubo una separación de $11 \mathrm{~mm}$. La vena renal se dividió en dos ramas, anterior y posterior, cuyos trayectos eran preaórtico y retro-aórtico, respectivamente.

\section{DISCUSIÓN}

El conocimiento de las variaciones arteriales es de importancia para clínicos, cirujanos e imagenólogos. A nivel renal, las arterias múltiples que se pueden presentar deben ser consideradas al momento de preparar pacientes para donaciones renales, para reconstrucciones vasculares o para efectuar nefrectomías radicales. Con el desarrollo de la tecnología de imágenes, es posible determinar a través de exámenes pre-operatorios la existencia de estos casos, lo que sin duda, evita lesiones iatrogénicas cuando se interviene la región.

Las variaciones del número de arterias renales ha sido descrito en porcentajes variables (25\%, Moore, $1984 ; 11,2 \%$, Wozniak, 2000; 18\%, Khamanarong et al., 2004; 20\%, Dhar $\&$ Lal, 2005). La literatura es rica en presentaciones de casos con más de una arteria renal por lado (Bulic et al., 1996; Loukas et al., 2005; Tanyeli et al., 2006; Deepthinath, 2006, entre otros).

Estas variaciones se pueden explicar a través de la embriología renal. Inicialmente los riñones se desarrollan en la pelvis, apareciendo alrededor de la quinta semana para luego ir ascendiendo hacia el abdomen. Su irrigación en la pelvis depende de ramas pélvicas de la aorta y a medida que asciende va siendo irrigado por vasos sucesivamente más altos, mientras que los vasos más bajos degeneran. Las variaciones vasculares resultan de la persistencia de vasos embrionarios que normalmente desaparecen cuando se forma la arteria renal definitiva. Las variaciones del riego sanguíneo son más comunes en los riñones ectópicos (Moore, 1984).

Las arterias renales han sido motivo de innumerables estudios sistemáticos, como también la presentación de casos con arterias dobles o triples, estas últimas en porcentajes bien bajos.

En un estudio realizado por Khamanarong et al. en 
267 cadáveres, observaron la disposición de dos arterias en $17 \%$ y de tres en $1 \%$. A pesar que este estudio demostró que más de dos arterias alcanzando el riñón es una disposición rara, se continua reportando casos aislados como el que han presentado recientemente Raheem et al. (2007) en un cadáver de un hombre de 89 años, que tenía tres arterias renales derechas originándose de la aorta, en donde la superior se originó por debajo del nivel de la arteria mesentérica superior y pasó por detrás de la vena cava inferior; la arteria renal media emergió de la línea media anterior de la aorta abdominal y pasó anterior a la vena cava inferior y la arteria renal inferior se originó de la parte antero-lateral de la aorta por debajo del nivel de emergencia de la arteria mesentérica inferior y pasó también por delante de la vena cava inferior. Los casos descritos en este trabajo difieren un poco del presentado por estos últimos autores, ya que la arteria renal inferior en ambos casos no tuvo un origen tan bajo, situándose por sobre la arteria mesentérica inferior. Las arterias renales las observamos en el lado izquierdo y se originaron desde la parte lateral de la aorta, teniendo un trayecto hacia el riñón de forma regular y escaleriforme en uno, a diferencia del otro caso, en que la arteria renal media (R2) tuvo un trayecto oblicuo e ingresó en el riñón por debajo de su hilio.

En presencia de arteria renales múltiples pueden ocurrir ligaduras accidentales de alguna de esas arterias y producir un infarto del correspondiente parénquima renal (Eitan et al., 2003). Por otra parte, alguna de estas arterias podrían comprimir la unión pélvico-ureteral o también el propio uréter, causando obstrucción al flujo urinario (Moore; Meng et al., 2002).

Así, es importante tener en cuenta la posibilidad de encontrar estas arterias, las que podrían complicar los procedimientos realizados en la región, siendo necesario realizar detallados angiogramas preoperatorios renales y aórticos, reduciendo así la dificultades técnicas de las cirugías respectivas.

OLAVE, E.; HENRÍQUEZ, J.; PUELMA, F.; CRUZAT, C. \& SOTO, A. Multiple renal arteries. Int. J. Morphol., 25(4):927-930, 2007.

SUMMARY: Due to increase of renal transplants and vascular reconstructions, the knowledge of variations of renal arteries is very important. The literature indicates that each kind have one artery, however sometimes there are origin and number variations. We describe in this article two cases with three arteries in the left side, found in the Anatomy Unit from Health Sciences Faculty, Universidad Católica del Maule, Talca, Chile. In one case, the origin of renal artery R1 (superior) from abdominal aorta, was to level of superior mesenteric artery origin. This R1 following a $30 \mathrm{~mm}$ trajectory divided in two minor branches of similar diameter and entered in the hilus passing in front of the renal vein. The origin of R2 (middle) was distal $4 \mathrm{~mm}$ to the R1, corresponding to a posterior branch that entered in the renal hilus and the R3 (inferior) originates distal $23 \mathrm{~mm}$ to the R2. This last artery entered in renal cortex, inferior to the renal sinus notch. In the second case, there were diferences with respect to the first case because R1 and R3 entered in the hilus, and, R2, with descendent course entered in renal cortex, similar to R3 of first case. The knowledge of these multiple arteries is necessary and should be kept in mind before and during surgery involving the renal region.

KEY WORDS: Anatomy; Kind; Renal arteries.

\section{REFERENCIAS BIBLIOGRÁFICAS}

Bulic, K.; Ivkic, G. \& Pavic T. A case of duplicated right renal artery and triplicated left renal artery. Ann Anat., 178(3):281-3,1996

Dangelo, J. G. \& Fattini, C. A. Anatomia Humana Sistêmica e Segmentar para o estudante de Medicina. $2^{\mathrm{a}}$ ed.Rio de Janeiro, Atheneu, 1988

Deepthinath, R.; Satheesha Nayak, B.; Mehta. R. B.; Bhat, S.; Rodriguez, V.; Samuel, V. P.; Venkataramana, V. \& Prasad AM. Multiple variations in the paired arteries of the abdominal arteries. Clin Anat., 19(6):566-8, 2006.

Dhar, P. \& Lal, K. Main and accessory renal arteries-a morphological study. Ital. J. Anat. Embryol., 110(2):10110,2005
Eitan, R.; Abu-Rustum, N. R.; Walter, J. L.; Barakat, R. R. Ligation o fan anatomic variant of renal vasculature during laparoscopic periaortic lymph node dissection: a cause of postoperative renal infarction. Gynecol. Oncol., 91(2):416-20.

Khamanarong, K; Prachaney, P.; Utraravichien, A; Tong-Un, T. \& Sripaoraya, K. Anatomy of renal arterial supply. Clin. Anat., 17(4):334-6, 2004.

Loukas, M.; Aparicio, S.; Beck, A.; Calderon, R. \& Kennedy, M. Rare case of right accessory renal artery originating as a common trunk with the inferior mesenteric artery: a case report. Clin. Anat., 18(7):530-5, 2005.

Meng, M. V.; Yeh, B. M.; Breiman, R. S.; Schwartz, B. F.; 
OLAVE, E.; HENRÍQUEZ, J.; PUELMA, F.; CRUZAT, C. \& SOTO, A. Arterias renales mútiples. Int. J. Morphol., 25(4):927-930, 2007

Coakley, F. V. \& Stoller, M. L.Precaval right renal artery: description and embryologic origin. Urology, 60(3): 402-5, 2002.

Moore, K. Embriologia Clínica. $3^{\text {a }}$ ed. Rio de Janeiro, Interamericana, 1984.

Moore, K. L. \& Dalley, A. F. Anatomía con Orientación Clínica. 4a Ed. Buenos Aires, Panamericana, 2002.

Raheem, O.; Glacken, P.; O’Brien, M.; Hickey, D. \& Mohan, P. A single male cadaver with multiple renal arteries. Ir. J. Med. Sci., 2007. (in press)

Tanyeli, E.; Uzel, M. \& Soyluoglu, A.I. Complex renal vascular variation: a case report. Ann. Anat., 188(5):4558,2006

Testut, L. \& Latarjet, A. Tratado de Anatomía Humana. Barcelona, Salvat, 1969.

Wozniak, W. T. Origin of the renal arteries from sides of aorta. Folia Morphol (Warsz)., 58(4):259-61, 2000

Williams, P. L.; Warwick, R.; Dyson, M. \& Bannister, L. H. Gray Anatomía. 37 ed. Rio de Janeiro, GuanabaraKoogan, 1995.
Dirección para correspondencia:

Prof. Dr. Enrique Olave Riffo

Facultad de Medicina

Universidad de La Frontera

Casilla 54-D

Temuco - CHILE

Email: eolave@ufro.cl

Recibido : 22-09-2007

Aceptado: 04-11-2007 